\title{
A grain size distribution model for non-catalytic gas-solid reactions
}

\author{
A.B.M. Heesink, W. Prins and W.P.M. van Swaaij \\ Department of Chemical Engineering, Twente University of Technology, PO Box 217, NL-7500 AE Enschede \\ (Netherlands)
}

(Received January 8, 1993; in final form June 4, 1993)

\begin{abstract}
A new model to describe the non-catalytic conversion of a solid by a reactant gas is proposed. This socalled grain size distribution (GSD) model presumes the porous particle to be a collection of grains of various sizes. The size distribution of the grains is derived from mercury porosimetry measurements. The measured pore size distribution is converted into a grain size distribution through a so-called pore-tosphere factor whose value is also derived from the porosimetry measurements. The grains are divided into a number of size classes. For each class the conversion rate is calculated either according to the shrinking core model, involving core reaction and product layer diffusion as rate-determining steps, or according to a new model in which some reaction at the grain surface is assumed to be limiting. The GSD model accounts for the phenomenon of pore blocking by calculating the maximum attainable conversion degree for each size class. In order to verify the model, two types of precalcined limestone particles with quite different microstructures were sulphided as well as sulphated. Furthermore, a single sample of sulphided dolomite was regenerated with a mixture of carbon dioxide and steam. For each reaction good agreement was attained between measured and simulated conversion vs. time behaviour.
\end{abstract}

\section{Introduction}

Gas-solid reactions are involved in many industrial processes. These reactions are generally of the type

$a \mathrm{~A}($ sol $)+b \mathrm{~B}$ (gas) $\longrightarrow c \mathrm{C}($ sol $)+d \mathrm{D}$ (gas)

Some examples are the removal of sulphur dioxide (hydrogen sulphide) by a solid sorbent in high temperature flue gas (coal gas) cleaning, the roasting of iron sulphide and the subsequent reduction of iron oxide to produce iron in steelworks and the calcination of limestone in the cement industry.

The microstructure of a porous solid affects its reactivity towards a reactant gas. Both diffusivity and kinetics depend on the microstructure, which may change during conversion. For instance, when the molar volume of the solid product exceeds that of the solid reactant, pore blocking might occur, resulting in zero diffusivity and cessation of conversion. Mathematical models for gas-solid reactions should include the influence of (changing) microstructure on particle diffusivity and volumetric kinetics. The term "volumetric kinetics" refers to the product of specific surface area and intrinsic kinetics, the latter reflecting the combined result of surface reaction kinetics and product layer diffusion. Many mathematical models have been developed. They can be roughly divided into two categories, namely grain models and pore models.

According to the grain model concept, a porous solid consists of small impervious pieces or grains that are dispersed in gas, which is then the continuous phase. Each grain is assumed to be converted according to the shrinking core model developed by Yagi and Kunii [1]. This concept was elaborated by Szekely and Evans [2], who derived relations to calculate the conversion $v s$. time behaviour of solids consisting of spherical, cylindrical or slab-like grains. The model of Szekely and Evans does not account for the change in grain size which occurs when the molar volumes of the solid reactant and solid product differ. Moreover, it is assumed that all grains are of equal size. Hartman and Coughlin [3] modified this model to simulate the sulphation of calcined limestone. They actually took into account the expansion of the calcium oxide grains during conversion. Later, Georgakis et al. [4] presented a similar changing grain size model. In these models all grains are still assumed to have initially the same size. Szekely and Propster [5] studied the effect of grain size distribution on the conversion behaviour of porous solids. Still assuming that grains do not 
change in size during conversion, they illustrated that a distribution in grain size greatly affects the particle conversion behaviour. Szekely and Propster regarded some statistical distributions and closed their publication with the statement "it would appear that when precise interpretation of the (conversion behaviour) measurements is required, in terms of the grain model, it would be desirable to complement the usual kinetic and structural measurements with a determination of the grain size distribution". More recently, Dam-Johansen et al. [6] developed a grain-micrograin model to describe the sulphation of calcium oxide. According to their model, the solid consists of porous grains which in turn are composed of impervious micrograins. The micrograins are converted by a shrinking, unreacted core mechanism until all free space inside a grain has been filled with product. Then the grains start to react according to the shrinking, partially reacted core mechanism. In this model both grains and micrograins are assumed to have initially the same size. Although the particle conversion behaviour can often be well described with an appropriate grain model, the dependence of surface area upon conversion is not well predicted [7]. Therefore Efthimiadis and Sotirchos [8] developed the partially overlapping grain model in which each grain is assumed to consist of a hard core, not overlapping with the cores of neighbouring grains, and a soft permeable shell which can overlap with the shells of other grains. By adjusting the ratio of core radius and grain radius, the development of surface area during conversion can be simulated.

In contrast with grain models, pore models regard the solid phase as the continuous phase. Various pore models have been developed, each describing pore tortuosity, pore interconnectivity and pore size distribution in its own way. Ramachandran and Smith [9] developed a single-pore model which describes pore diffusion, diffusion through a product layer being precipitated on the pore walls, and surface reaction within a single cylindrical pore. The phenomenon of pore mouth closure is included as well. Ramachandran and Smith assumed all pores to have initially the same size. Later models took into account the distribution in pore size [10-13] and the interconnectivity of pores [14]. Good results have been obtained with these more complex models where the influence of pore size distribution was clearly shown (e.g. by Sotirchos and Zarkanitis [15]).

A new model is now proposed. This grain size distribution (GSD) model is based on the grain model of Szekely and Evans [2], but additionally accounts for a measurable distribution in grain size, changing grain size and the possibility of pore blocking. This is done by converting the measured pore size distribution into a grain size distribution through a newly defined "pore-to-sphere" factor. Pore blocking is assumed to occur when the expansion of grains from a certain grain size class equals the volume of pores from the corresponding pore size class. The GSD model is meant to describe the volumetric kinetics of solid particles while accounting for the effects of pore-grain size distribution and pore blocking. Unlike in (partially) overlapping grain models, the grains are assumed to be unconsolidated and non-overlapping. Thus, although the evolution of volumetric kinetics during conversion can be well described, the GSD model will not predict the evolution of surface area during conversion very well. Furthermore, intraparticle transport is not considered: it is assumed that the gas phase inside a particle is homogeneous in nature. The model should therefore only be applied to particles which are converted in the kinetically controlled regime. However, it can be modified in this respect by combination with a proper pore model. This will be illustrated in a future paper.

It is important to note that the present model cannot be used to describe the conversion behaviour of particles in which fresh pores (and grains) develop during conversion. However, when the formation of pores (and grains) only takes place during the initial stage of conversion (nucleation stage), the GSD model may be applied to describe conversion $v s$. time behaviour after the nucleation stage. This approach is similar to that of the crackling grain model developed by Park and Levenspiel [16].

First, the shrinking core model will be discussed. This classical model, which discriminates between product layer diffusion and reaction at the surface of the unreacted core (core reaction) as possible rate-determining steps, will be extended by a third step, namely reaction at the surface of the grain (grain reaction). Second, it will be shown how the grain size distribution is derived from a mercury porosigram by applying the concept of a pore-tosphere factor. Finally, the GSD model will be verified experimentally. The calculated conversion vs. time behaviour will be compared with the measured behaviour during the sulphation and sulphidation of two types of precalcined limestone particles with different microstructures. This will also be done for the regeneration of sulphided dolomite particles with a mixture of steam and carbon dioxide. The GSD model is used to establish the governing mechanisms of the reactions involved. 


\section{Shrinking core model}

The classical model is illustrated in Fig. 1, where a sketch of a partly converted sphere is linked up with a diagram showing the corresponding radial profile of the involved gaseous reactant. The outer shell of the spherical particle consists of solid product only, whereas the core contains the original solid reactant. Core and product layer materials do not mix, implying that there is a sharp interface between core and product layer. Two different concentration profiles are shown, one referring to the extreme case of conversion fully controlled by core reaction, the other to the extreme case of conversion fully controlled by product layer diffusion. Below, each extreme is worked out. Afterwards, a third possible limiting step, namely grairt reaction, is considered.

\subsection{Core reaction limitation}

In the case of core reaction limitation the conversion rate of a grain with initial radius $R_{\mathrm{o}}$ can be calculated from

$\frac{4}{3} \pi R_{\mathrm{o}}^{3} N_{\mathrm{o}} \frac{\mathrm{d} X}{\mathrm{~d} t}=4 \pi R_{\mathrm{c}}^{2} k_{\mathrm{c}} C_{\mathrm{c}}$

$R_{\mathrm{c}}$ represents the radius of the unconverted core, which can be calculated according to

$R_{\mathrm{c}}=(1-X)^{1 / 3} R_{\mathrm{o}}$

The parameter $k_{\mathrm{c}}$ represents the kinetic constant of the surface reaction at the core, which is assumed to be of first order in the gaseous reactant. $C_{c}$ represents the concentration of the gaseous reactant at the core surface, which in the case of core reaction limitation equals the concentration at the grain surface, $C_{\mathrm{g}}$. The parameter $N_{\mathrm{o}}$ represents the initial concentration of the solid reactant in the grain and depends on the purity of the grain $(P)$ and the molecular volume of the solid reactant:

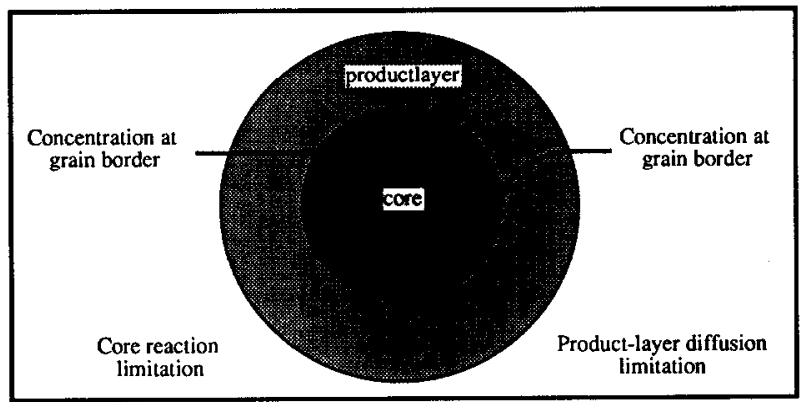

Fig. 1. Illustration of the shrinking core model for the conversion of a single grain. Concentration profiles: left-hand side, core reaction limitation; right-hand side, product layer diffusion limitation.
$N_{\mathrm{o}}=\frac{P}{V_{\text {sol ,reac }}}$

It is assumed here that the density of impurities in the grain is equal to that of the solid reactant.

Combination of eqns. (1) and (2), while substituting $C_{\mathrm{c}}$ by $C_{\mathrm{g}}$, yields

$\frac{\mathrm{d} X}{\mathrm{~d} t}= \begin{cases}3 K_{\mathrm{c}}(1-X)^{2 / 3} & \text { for } X<X_{\max } \\ 0 & \text { for } X=X_{\max }\end{cases}$

$K_{\mathrm{c}}$ is some kind of overall reaction rate constant (in the case of core reaction limitation) and is given by

$K_{\mathrm{c}}=\frac{k_{\mathrm{c}} C_{\mathrm{g}}}{R_{\mathrm{o}} N_{\mathrm{o}}}$

The value of the maximum attainable conversion $X_{\max }$ is either unity or less than unity when the possibility of grain expansion is limited. Later on it will be demonstrated how $X_{\max }$ is to be calculated.

\subsection{Product layer diffusion limitation}

In the case where product layer diffusion is rate controlling, the conversion rate can be calculated from

$\frac{4}{3} \pi R_{\mathrm{o}}^{3} N_{\mathrm{o}} \frac{\mathrm{dX}}{\mathrm{d} t}=4 \pi R^{2} \mathrm{D}_{\mathrm{s}}\left(\frac{\mathrm{d} C}{\mathrm{~d} r}\right)_{r=R}$

$D_{\mathrm{s}}$ represents the diffusivity of the product layer and $r$ is the distance between the centre of the grain and the radial position $R$ in the product layer. Since no reaction takes place in the product layer, the flow of gaseous reactant through the product layer does not vary with radius and eqn. (6) may be rewritten as

$\frac{\mathrm{d} X}{\mathrm{~d} t}=\frac{3 D_{\mathrm{s}}}{N_{\mathrm{o}} R_{\mathrm{o}}^{3}}\left(\frac{C_{\mathrm{g}}-C_{\mathrm{c}}}{\frac{1}{R_{\mathrm{c}}}-\frac{1}{R_{\mathrm{g}}}}\right)$

The radius of a partly converted grain can be calculated from

$R_{\mathrm{g}}=R_{\mathrm{o}}(K X+1)^{1 / 3}$

where $K$ represents the expansion factor given by

$K=N_{\mathrm{o}}\left(V_{\text {sol, prod }}-V_{\text {sol, reac }}\right)$

$C_{\mathrm{c}}$ will be much smaller than $C_{\mathrm{g}}$ when product layer diffusion fully controls conversion. This and additional substitution of $R_{\mathrm{c}}$ and $R_{\mathrm{g}}$ according to eqns. (2) and (8) yields 


$$
\frac{\mathrm{d} X}{\mathrm{~d} t}= \begin{cases}\frac{3 K_{\mathrm{p}}}{\left(\frac{1}{1-X}\right)^{1 / 3}-\left(\frac{1}{1+K X}\right)^{1 / 3}} & \text { for } X<\mathrm{X}_{\max } \\ 0 & \text { for } X=X_{\max }\end{cases}
$$

The corresponding overall reaction rate constant (in the case of product layer diffusion control) $K_{\mathrm{p}}$ is then

$K_{\mathrm{p}}=\frac{D_{\mathrm{s}} C_{\mathrm{g}}}{N_{\mathrm{o}} R_{\mathrm{o}}^{2}}$

The overall reaction rate constants $K_{\mathrm{c}}$ and $K_{\mathrm{p}}$ as given by eqns. (5) and (11) respectively appear to be interrelated according to

$K_{\mathrm{p}}=\frac{K_{\mathrm{c}}}{\psi}$ with $\psi=\frac{R_{\mathrm{o}} k_{\mathrm{c}}}{D_{\mathrm{s}}}$

It should be noted that eqn. (10) cannot be used to calculate the initial conversion vs. time behaviour, i.e. when no product layer has been built up yet. Use of eqn. (10) would yield an infinitely high initial conversion rate. Here it is assumed that the core reaction limits the initial conversion rate. Therefore a general expression including both core reaction and product layer diffusion as possible steps is derived below.

\subsection{General expression according to the classical shrinking core model}

Combining eqns. (1) and (7), while substituting $R_{\mathrm{c}}$ and $R_{\mathrm{g}}$ according to eqns. (2) and (8), and introducing $K_{\mathrm{c}}$ and $\psi$ as defined by eqns. (5) and (12) results in a general expression for the conversion rate from which $C_{c}$ has been eliminated. This expression is valid for both core reaction control and product layer diffusion control as well as for intermediate cases.

$\frac{\mathrm{d} X}{\mathrm{~d} t}=$

$\begin{cases}\frac{3 K_{\mathrm{c}}}{\frac{1}{(1-X)^{2 / 3}}+\psi\left(\frac{1}{(1-X)^{1 / 3}}-\frac{1}{(1+K X)^{1 / 3}}\right)} & \text { for } X<X_{\max } \\ 0 & \text { for } X=X_{\max }\end{cases}$

Obviously for large values of $\psi$ this expression reduces to eqn. (10) representing the case of product layer diffusion control. At $\psi$ values close to zero eqn. (13) turns into eqn. (4) which is valid in the case of core reaction control. The value of $\psi$ thus determines which mechanism is rate controlling:

$\psi \ll 1$ : core reaction limitation

$\psi \gg 1$ : product layer diffusion limitation

Note that eqn. (13) meets the demand of a limited initial conversion rate:

$\operatorname{Lim}_{X \rightarrow 0}\left(\frac{\mathrm{d} X}{\mathrm{~d} t}\right)=3 K_{\mathrm{c}}(1-X)^{2 / 3}$

The time needed to obtain a certain conversion can be derived by integration of eqn. (13):

$$
\begin{aligned}
t(X)= & \frac{1-(1-X)^{1 / 3}}{K_{\mathrm{c}}}+\frac{\psi}{2 K_{\mathrm{c}}} \\
& \times\left(1-(1-X)^{2 / 3}+\frac{1}{K}\left[1-(K X+1)^{2 / 3}\right]\right) \\
& \text { for } X<X_{\max }
\end{aligned}
$$

\subsection{Grain reaction limitation}

Equations (13) and (16) are valid when core reaction and product layer diffusion are the only rate-determining steps in the conversion mechanism. However, it is not unlikely that the gaseous reactant, which has to pass the product layer by solid state (counter)diffusion, is first converted into some ionic species at the outer surface of a grain according to some grain reaction. Therefore we consider the grain reaction as a third rate-determining step that may limit the conversion rate.

If some grain reaction governs the conversion rate, the following applies:

$\frac{4}{3} \pi R_{\mathrm{o}}^{3} N_{\mathrm{o}} \frac{\mathrm{d} X}{\mathrm{~d} t}=4 \pi R_{\mathrm{g}}^{2} k_{\mathrm{g}} C_{\mathrm{g}}$

Combining eqns. (17) and (8), together with some rearrangement yields

$\frac{\mathrm{d} X}{\mathrm{~d} t}= \begin{cases}3 K_{\mathrm{g}}(K X+1)^{2 / 3} & \text { for } X<X_{\max } \\ 0 & \text { for } X=X_{\max }\end{cases}$

with $K_{\mathrm{g}}$ representing the overall reaction rate constant (in the case of grain reaction limitation)

$K_{\mathrm{g}}=\frac{k_{\mathrm{g}} C_{\mathrm{g}}}{R_{\mathrm{o}} N_{\mathrm{o}}}$

Integration of eqn. (18) leads to 
$X(t)= \begin{cases}\frac{\left(K K_{\mathrm{g}} t+1\right)^{3}-1}{K} & \text { for } X<X_{\max } \\ X_{\max } & \text { for } X=X_{\max }\end{cases}$

From eqn. (18) it follows that the conversion rate increases as conversion proceeds and actually reaches its maximum value at complete conversion, i.e. when the grain has its highest surface area. Although the phenomenon of an increasing conversion rate during the first part of a conversion process has been observed in practice (e.g. by Tseng and Edgar [17] during the combustion of coal char), such behaviour has never been observed during the final part of conversion. This probably explains the fact that grain reaction limitation is not considered in the classical shrinking core model. However, as will be shown below, grain reaction limitation can lead to "normal" conversion vs. time behaviour when the grain size distribution is taken into account. Therefore grain reaction limitation is considered here as a third possible mechanism.

It is important to note that it is not possible to couple eqns. (13) and (18) by elimination of $C_{\mathrm{g}}$. The parameter $C_{\mathrm{g}}$ used in eqn. (13) (by means of $K_{\mathrm{c}}$ ) refers to the concentration of the (ionic) species which is formed at the grain surface, diffuses through the product layer and subsequently reacts at the core surface. The parameter $C_{\mathrm{g}}$ in eqn. (18) (through $K_{q}$ ) refers to the concentration of the adsorbed gaseous reactant at the grain surface. In general it is not possible to combine the three steps of grain reaction, product layer diffusion and core reaction without violating the assumption of the classical shrinking core model that the concentration of the gaseous reactant (i.e. ionic species) at the grain surface remains constant during conversion. Therefore, when simulating measured conversion $v$ s. time behaviour with the GSD model, we will only apply the three extreme cases of grain reaction limitation (eqn. (20)), product layer diffusion limitation (eqn. (16) with $\psi \gg 1$ ) and core reaction limitation (eqn. (16) with $\psi=0$ ). Intermediate cases will not be considered in order to avoid violation of the classical shrinking core model and to reduce the number of model fit parameters $\left(k_{\mathrm{g}}, k_{\mathrm{c}}\right.$ and $\left.D_{\mathrm{s}}\right)$ applied.

\section{Grain size distribution model}

According to the GSD model, a porous particle is regarded as a collection of small spherical grains of various sizes. Figure 2 illustrates this concept for a calcined limestone particle, which has a bidisperse nature according to the literature $[6,18]$.

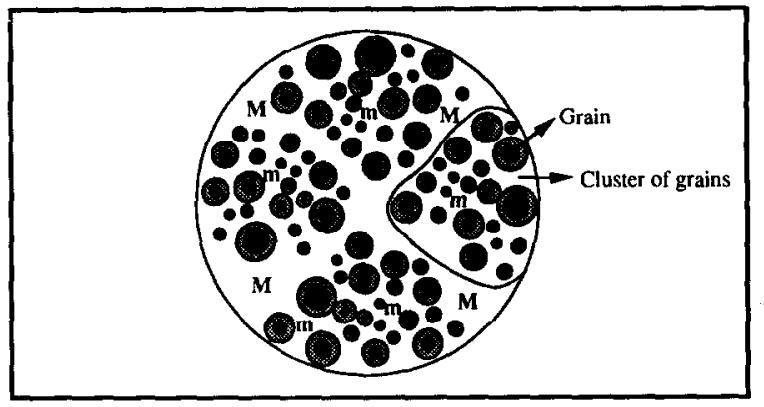

Fig. 2. Representation of the GSD model concept for a partly converted bidisperse particle: $M$, macropores between clusters; $\mathrm{m}$, micropores within clusters, between grains.

The grains are gathered in clusters. Micropores are located within these clusters whereas macropores are located in between the clusters. It should be noted that the GSD model is not only applicable to bidisperse particles. Basically, the conversion $v s$. time behaviour of all types of particles, including of course simple monodisperse ones, can be described.

Frevel and Kressley [19] studied mercury porosigrams of dense packings of microspheres with radii between 120 and $180 \mu \mathrm{m}$. They concluded that the apparent pore size within these packings is determined by the size of the spheres only. For a packing of uniform microspheres a value of three is found for the ratio of sphere radius and pore radius. This ratio is referred to as the "pore-tosphere factor" $F$. For a mixture of different size spheres the porosimetry curve obtained appears to be very similar to a smoothed composite of the porosimetry curves measured for grains from the individual size classes. In this case the value of the pore-to-sphere factor depends on the size distribution of the spheres and the mode of packing but is always smaller than three.

In the present work the findings of Frevel and Kressley [19] are used to derive the sizes of the grains inside a porous solid from mercury porosimetric measurements. To verify whether this is allowed for spheres with sizes typical for grains inside a porous solid (nanometre range), mercury porosimetry was performed on a test sample of calcined limestone particles which were also studied by means of scanning electron microscopy (SEM). Figure 3(a) and 3(b) show respectively the mercury porosigram and the SEM photograph on which spherical grains of various sizes can clearly be distinguished. The measured pore radius ranges from about 7 to $40 \mathrm{~nm}$ whereas the average pore radius amounts to about $17 \mathrm{~nm}$. The radii of some 70 grains were determined. The radii range from about 

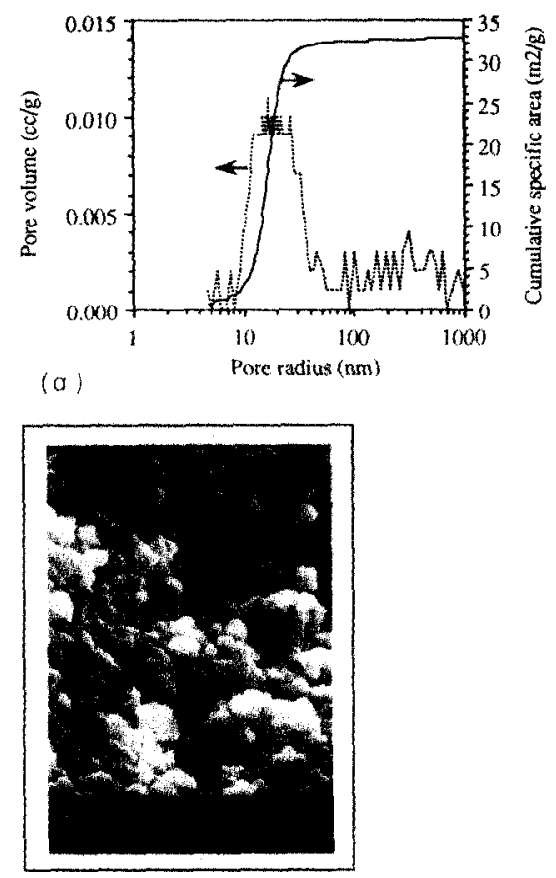

(b)

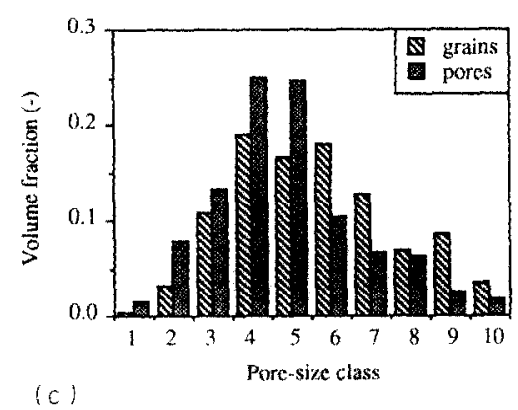

Fig. 3. Verification of the relationship between grain radius and pore radius by comparing (a) a mercury porosigram and (b) an SEM photograph of a test sample of calcined limestone particles. Value of pore-to-sphere factor used in (c) is 1.8. Pore size classes in (c): $1,6-8 ; 2,9-12 ; 3,13-15 ; 4,16-18 ; 5$, $19-21 ; 6,22-24 ; 7,25-27 ; 8,28-30 ; 9,31-33 ; 10,34-36$ nm.

12 to $40 \mathrm{~nm}$, the average grain radius being some $30 \mathrm{~nm}$. When comparing the average pore radius with the average grain radius, a pore-to-sphere factor of $30 / 17=1.8$ can be calculated. After dividing the measured grain radii by the value of 1.8 , both pores and grains were divided into 10 size classes. In Fig. 3 (c) the volume fractions of the various pore and grain classes are plotted. The agreement between pore and grain size distributions justifies the conclusion that a grain size distribution can indeed be derived from mercury porosimetric measurements.

It will now be shown how the actual value of the pore-to-sphere factor can be derived from a mercury porosigram. The mercury porosigram is first divided into a limited number $(N)$ of pore radius intervals. Figure 4 shows how this is done. Since particle reactivity is proportional to the amount of specific surface area involved, a criterion is available for deciding which part of the porosigram should be taken into account. In the case of Fig. 4 pores with a radius larger than about $110 \mathrm{~nm}$ (representing only a minor part of the total specific surface area) are neglected. These pores are considered to be macropores which are located between clusters of grains. Therefore the sizes of the macropores are assumed to correspond to the sizes of clusters rather than grains. According to the definition of the poreto-sphere factor, the grain radius that corresponds to the pore radius of a certain interval is given by

$R_{\mathrm{o}, i}=F R_{\mathrm{p}, i}$

The pore volume for interval $i, V_{\mathrm{p}, i}$, is a measure of the total weight of grains with radius $R_{\mathrm{o}, i}$. The weight fraction of grains belonging to size class $i$, $\nu_{i}$, can thus be calculated from

$\nu_{i}=\frac{V_{\mathrm{p}, i}}{\sum_{j=1}^{j=N} V_{\mathrm{p}, j}}$

The specific surface area $A$ of particles containing different size spheres can be obtained from

$A=\frac{3}{\rho_{\mathrm{sol}, \text { reac }}} \sum_{i=1}^{i=N} \frac{\nu_{i}}{R_{\mathrm{o}, i}}$

Thus, once the specific surface area of the solid reactant is known, the value of the pore-to-sphere factor can be derived from

$F=\frac{3}{A \rho_{\mathrm{sol}, \text { reac }}} \sum_{i=1}^{i=N} \frac{\nu_{i}}{R_{\mathrm{p}, i}}$

The value of $A$ can be measured in several ways, but also by mercury porosimetry. Consequently, a

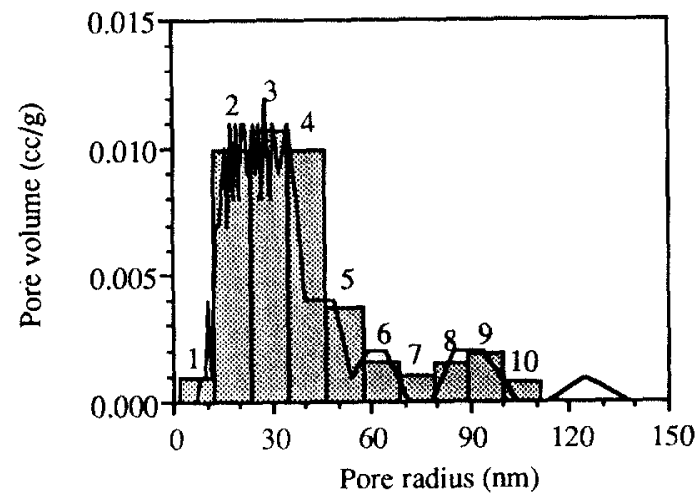

Fig. 4. Division of a mercury porosigram into intervals (here 10) according to the GSD model. 
single mercury porosimetry measurement is sufficient to determine the value of the pore-to-sphere factor and subsequently the grain size distribution.

With the known values for $R_{\mathrm{o}, i}$ the conversion of each grain class, $X_{i}$, can be calculated as a function of time using either (the inverse of) eqn. (16) when core reaction or product layer diffusion is the governing mechanism or eqn. (20) in the case of grain reaction limitation. In the former case calculations have to be performed numerically, since eqn. (16) cannot be inverted in an analytical way. For proper use of eqns. (16) and (20) the maximum attainable conversion of each grain class, $X_{\max , i}$, must be known. In our model $V_{\mathrm{p}, i}$ is assumed to represent the volume available for the expansion of grains from size class $i$. Then the value of $X_{\max , i}$ can be calculated from $V_{\mathrm{p}, i}$ and the expansion factor $K$, which is a measure of the difference between the molar volumes of solid reactant and solid product:

$X_{\max , i}=\frac{\rho_{\mathrm{sol}, \text { reac }} V_{\mathrm{p}, i}}{K \nu_{i}} \quad\left(\right.$ if $<1$, else $\left.X_{\max , i}=1\right)$

An important assumption of our model is that the access to grains of a certain size class is not disturbed when grains of other size classes have reached maximum conversion and their corresponding pores have become blocked.

Finally, the total conversion of a particle is given by

$X_{\text {tot }}=\sum_{i=1}^{i=N} \nu_{i} X_{i}$

\section{Experimental details}

The GSD model has been tested by comparing several experimentally determined conversion vs. time curves with predicted ones. All conversion experiments were carried out by measuring the change in sample weight during reaction using thermogravimetric (TG) analysis. Three reactions of importance in the regenerative high temperature desulphurization process being developed at our university were investigated.

1. Sulphidation of precalcined limestone:

$\mathrm{CaO}+\mathrm{H}_{2} \mathrm{~S} \longrightarrow \mathrm{CaS}+\mathrm{H}_{2} \mathrm{O}$

2. Sulphation of precalcined limestone:

$\mathrm{CaO}+\mathrm{SO}_{2}+\frac{1}{2} \mathrm{O}_{2} \longrightarrow \mathrm{CaSO}_{4}$

3. Regeneration of sulphided dolomite:

$\mathrm{CaS} . \mathrm{MgO}+\mathrm{CO}_{2}+\mathrm{H}_{2} \mathrm{O} \longrightarrow \mathrm{CaCO}_{3} \cdot \mathrm{MgO}+\mathrm{H}_{2} \mathrm{~S}$

\subsection{Sulphidation and sulphation}

Sulphidation and sulphation experiments were carried out in an atmospheric thermogravimetric analyser (TGA, type Setaram TG-85) using two types of limestone. The first (Wülfrath) is a natural limestone which did not undergo any pretreatment. The second (Lhoist agglomerate) consists of particles which were agglomerated from limestone powder (75 wt.\% of powder particles smaller than $75 \mu \mathrm{m}$, 25 wt.\% smaller than $150 \mu \mathrm{m}$ ) by applying $2 \mathrm{wt} . \%$ bentonite cement. Table 1 gives the compositions of the materials used.

A sample of about $1.5 \mathrm{~g}$ (particle diameters between 106 and $212 \mu \mathrm{m}$ ) of each limestone type was calcined in the TGA at $800{ }^{\circ} \mathrm{C}$ while flushing with helium:

$\mathrm{CaCO}_{3} \longrightarrow \mathrm{CaO}+\mathrm{CO}_{2}$

After about $15 \mathrm{~min}$ no further decrease in weight was observed and calcination was stopped. About $1 \mathrm{~g}$ of the calcined material was set aside for mercury porosimetry measurements (porosimeter Carlo Erba Strumentazione, DRU model 204). The remainder was used for the sulphidation and sulphation experiments.

Figure 5 shows that the porosigram of the calcined Lhoist agglomerate particles has two peaks, whereas only one peak has been found for the Wülfrath particles. Because of this pronounced difference in microstructure, the two selected particle types are quite suitable to test the GSD model. The specific surface area derived from the mercury porosimetry measurements amounts to $21.5 \mathrm{~m}^{2} \mathrm{~g}^{-1}$ for the Wülfrath particles and $25.3 \mathrm{~m}^{2} \mathrm{~g}^{-1}$ for the Lhoist agglomerate particles. Most of the surface area of the Wülfrath particles corresponds to pores smaller than $100 \mathrm{~nm}$. Accordingly, pores with a radius larger

TABLE 1. Chemical compositions (wt.\%) of the limestone and dolomite particles used for experimental verification of the GSD model

\begin{tabular}{lccr}
\hline Component & $\begin{array}{l}\text { Wülfrath } \\
\text { natural }\end{array}$ & $\begin{array}{l}\text { Lhoist } \\
\text { agglomerate }\end{array}$ & $\begin{array}{l}\text { Lhoist } \\
\text { dolomite }\end{array}$ \\
\hline $\mathrm{CaCO}_{3}$ & 97.10 & 94.90 & 54.90 \\
$\mathrm{MgCO}_{3}$ & 0.90 & 1.32 & 44.70 \\
$\mathrm{Si}$ & 0.80 & 1.10 & 0.04 \\
$\mathrm{Fe}$ & 0.17 & 0.15 & 0.36 \\
$\mathrm{Al}$ & $<0.10$ & $<0.10$ & $<0.01$ \\
$\mathrm{~S}$ & $<0.10$ & $<0.10$ & $<0.01$ \\
$\mathrm{~K}$ & $<0.10$ & $<0.10$ & $<0.01$ \\
$\mathrm{Sr}$ & $<0.01$ & $<0.01$ & $<0.01$ \\
$\mathrm{Na}$ & $<0.01$ & 0.03 & $<0.01$ \\
$\mathrm{CaO}$ content & 95.72 & 92.34 & 58.64 \\
after calcination & & & \\
\hline
\end{tabular}



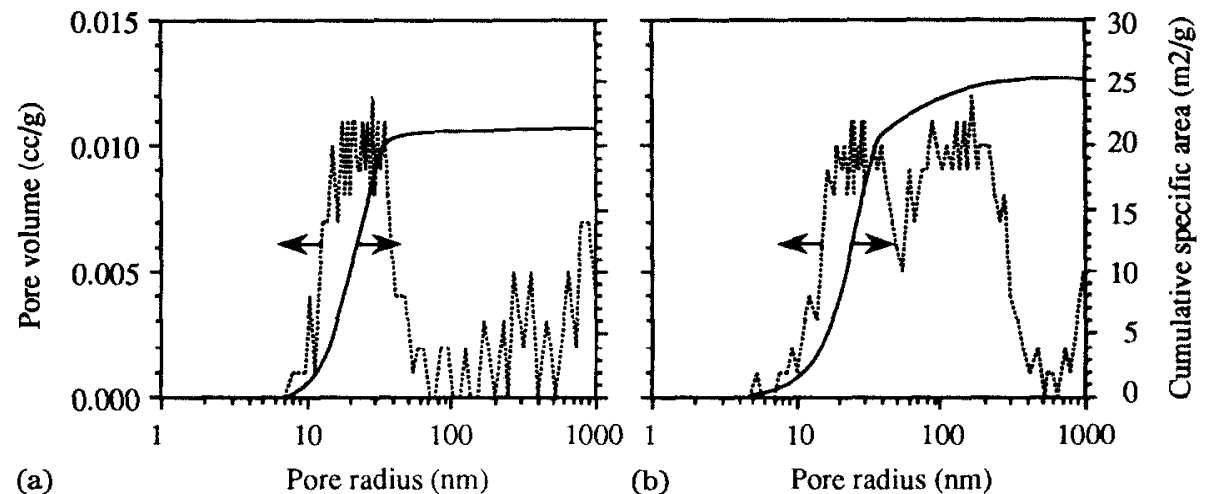

Fig. 5. Mercury porosigrams of calcined limestone particles: (a)

than $100 \mathrm{~nm}$ are considered as macropores located between clusters of grains. For the Lhoist agglomerate particles this boundary between micro- and macropores is located at approximately $200 \mathrm{~nm}$.

The observed weight change during the TGA experiments should only reflect the reaction kinetics of the sample. In order to minimize the influence of mass and heat exchange between the sample basket and gas atmosphere, the amount of sample used for the sulphidation and sulphation experiments was kept small (approximately $3 \mathrm{mg}$ ). Differential operation was assured by the combined use of a small amount of sample and a sufficiently high gas flow rate through the TGA, i.e. $400 \mathrm{ml}$ (STP) $\mathrm{min}^{-1}$.

The sulphation reaction was carried out at 700 ${ }^{\circ} \mathrm{C}$. The feed gas mixture contained 0.2 vol. $\%$ sulphur dioxide, 9.8 vol.\% nitrogen, 2 vol.\% oxygen and balance helium. Sulphidation experiments were also carried out at $700{ }^{\circ} \mathrm{C}$ while using a gas mixture of 2 vol.\% hydrogen sulphide, 4 vol.\% hydrogen (to stabilize the hydrogen sulphide) and balance helium. Before adding the hydrogen sulphide to the gas flow, the hydrogen-helium mixture was passed through a bed (heated to $85^{\circ} \mathrm{C}$ ) of oxygen-binding copper catalyst (type BASF R 3-11) to remove small quantities of oxygen originally present in the bottle gas. This was done because experiments had shown that even the presence of 5 ppm oxygen by volume in the gas mixture leads to significant oxidation of the calcium sulphide product towards calcium sulphate. 'The formation of calcium sulphate results in misinterpretation of the measured conversion vs. time behaviour as a consequence of the large difference between the molecular weights of calcium sulphate $(M=136)$ and calcium sulphide $(M=72)$.

All required gases were taken from bottles. The composition of the applied gas mixtures was controlled by means of calibrated electronic mass flow controllers.

\subsection{Ad regeneration}

Prior to regeneration, about $600 \mathrm{mg}$ of Lhoist dolomite particles (diameters between 106 and 212 $\mu \mathrm{m}$ ) were fully calcined in a high pressure TGA (HP-TGA, constructed by DMT). Table 1 gives the composition of the applied Lhoist dolomite particles. Calcination was performed under nitrogen at a temperature of $700^{\circ} \mathrm{C}$ and at atmospheric pressure:

$\mathrm{CaCO}_{3} \cdot \mathrm{MgCO}_{3} \longrightarrow \mathrm{CaO} \cdot \mathrm{MgO}+2 \mathrm{CO}_{2}$

Calcination was stopped after about $4 \mathrm{~h}$ when no further decrease in weight was observed. About 300 $\mathrm{mg}$ of the obtained $\mathrm{CaO} . \mathrm{MgO}$ particles were used for mercury porosimetry. Figure 6 shows the obtained porosigram. This porosigram was used indirectly to obtain the size distribution of the CaS.MgO grains. This method was chosen because direct mercury porosimetry of the sulphided particles had been shown to be disturbed by the fast oxidation of $\mathrm{CaS} . \mathrm{MgO}$ towards $\mathrm{CaSO}_{4} . \mathrm{MgO}$ occurring when the CaS.MgO sample was taken out of the HP-TGA and contacted with air. First the size distribution of the CaO.MgO grains was derived in the way described above using the porosigram of Fig. 6 . The boundary between micro- and macropores was

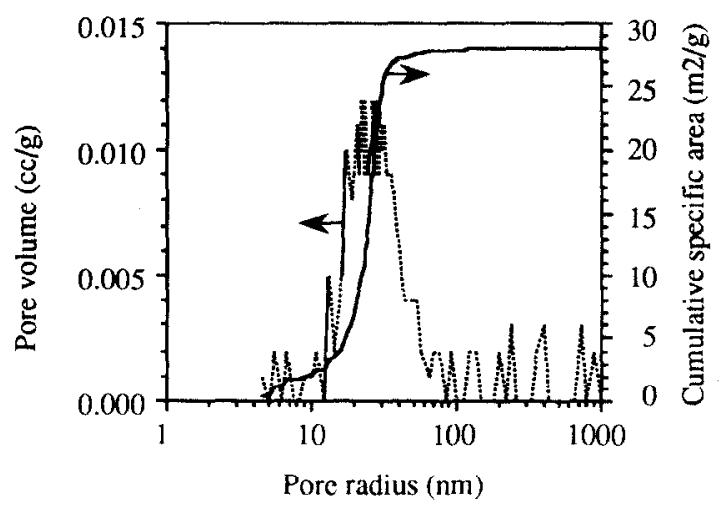

Fig. 6. Mercury porosigram of fully calcined dolomite particles (Lhoist dolomite). 
fixed at $120 \mathrm{~nm}$. Then the obtained distribution was transformed into the size distribution of the CaS.MgO grains by use of eqn. (8) $(K=0.38)$.

The remaining $25 \mathrm{mg}$ of fully calcined dolomite not used for mercury porosimetry were sulphided in the HP-TGA at $600{ }^{\circ} \mathrm{C}$ and atmospheric pressure while using a gas mixture consisting of 2 vol.\% hydrogen sulphide, 18 vol.\% hydrogen and balance nitrogen:

$\mathrm{CaO} . \mathrm{MgO}+\mathrm{H}_{2} \mathrm{~S} \longrightarrow \mathrm{CaS} . \mathrm{MgO}+\mathrm{H}_{2} \mathrm{O}$

The required gases were taken from bottles. Any traces of oxygen were removed from the nitrogen stream in the same way as described above for the atmospheric TGA. Sulphidation was stopped after some $30 \mathrm{~min}$ when no further change in weight was observed.

After sulphidation was completed the conditions were adjusted for regeneration. During adjustment the sample was flushed with helium. Regeneration was carried out at a temperature of $600{ }^{\circ} \mathrm{C}$ and a pressure of $10 \mathrm{bar}$. The applied gas mixture consisted of 20 vol.\% carbon dioxide, 20 vol.\% steam and balance nitrogen. The total flow rate was fixed at 51 (STP) $\min ^{-1}$. The steam was obtained by evaporating a small stream of water which was supplied by an HPLC pump. Again, any traces of oxygen were previously removed from the nitrogen stream. The composition of the applied gas mixtures was controlled by means of calibrated electronic mass flow controllers.

\section{Results and discussion}

In this section the results of TGA measurements will be compared with GSD model predictions. Experimentally determined conversion $v s$. time curves will be simulated by model computations in which either product layer diffusion, core reaction or grain reaction is assumed to be rate controlling. Intermediate situations will not be considered.

As explained above, use of the GSD model requires a mercury porosigram to calculate the pore-to-sphere factor and subsequently the size distribution of the grains. Figures 5 and 6 have been used for the present calculations. The Wülfrath porosigram was divided into 36 , the Lhoist porosigram into 53 and the Lhoist dolomite porosigram into 47 pore size classes. The largest pore radii involved are $125 \mathrm{~nm}$ (Wülfrath), $203 \mathrm{~nm}$ (Lhoist agglomerate) and 123 nm (Lhoist dolomite). Larger pores do not represent a significant surface area and are regarded as macropores. Values of 1.88 (Wülfrath), 1.04 (Lhoist agglomerate) and 1.34 (Lhoist dolomite) have been derived for the pore-to-sphere factors. As already stated above, the grain size distribution derived from the porosigram of the fully calcined Lhoist dolomite particles (Fig. 6) was transformed into the corresponding grain size distribution of the sulphided particles by making use of eqn. (8) with a $K$ value of 0.38 . Accordingly, the radii of the fully calcined grains were multiplied by 1.113 to obtain the corresponding radii of the sulphided grains.

In the following presentation of results, values of the model fit parameters $k_{\mathrm{c}}, D_{\mathrm{s}}$ and $k_{\mathrm{g}}$ have dimensions corresponding to volumetric dimensions $\left(\mathrm{mol} \mathrm{m} \mathrm{m}^{-3}\right)$ of $C_{\mathrm{g}}$. In the case of sulphation and sulphidation $C_{\mathrm{g}}$ refers to the volumetric (bulk) concentrations of sulphur dioxide and hydrogen sulphide respectively. Then the parameters $k_{\mathrm{c}}$ and $k_{\mathrm{g}}$ have dimensions of $\mathrm{m} \mathrm{s}^{-1}$ and $D_{\mathrm{s}}$ has dimensions of $\mathrm{m}^{2} \mathrm{~s}^{-1}$. In the case of regeneration $C_{\mathrm{g}}$ refers to the product of the volumetric (bulk) concentrations of carbon dioxide and water. Then the parameters $k_{\mathrm{c}}$ and $k_{\mathrm{g}}$ correspondingly have dimensions of $\mathrm{m}^{4}$ $\mathrm{mol}^{-1} \mathrm{~s}^{-1}$ and $D_{\mathrm{s}}$ has dimensions of $\mathrm{m}^{5} \mathrm{~mol}^{-1} \mathrm{~s}^{-1}$. It should be noted, however, that the GSD model can also be applied when an adsorption-desorption equilibrium exists at the grain surface. In that case $C_{\mathrm{g}}$ would represent a surface concentration and $k_{\mathrm{c}}$, $k_{\mathrm{g}}$ and $D_{\mathrm{s}}$ would have the corresponding dimensions.

\subsection{Sulphation}

Figure 7 shows conversion vs. time diagrams in which the measured and calculated best-fit curves for the sulphation experiments are plotted. As a result of the large difference between the molar volumes of calcium oxide and calcium sulphate $\left(1.68 \times 10^{-5}\right.$ and $5.22 \times 10^{-5} \mathrm{~m}^{3} \mathrm{~mol}^{-1}$ respectively), pore blocking occurs and total conversion cannot be achieved. The maximum possible sulphation degree of Lhoist agglomerate particles (0.6) appears to be much larger than that of Wülfrath particles (0.4), which can be explained by their difference in microporosity [20] (see also Fig. 5).

The GSD fits of both measured conversion vs. time curves are quite satisfactory in the case where product layer diffusion is assumed to be the governing mechanism. The GSD model is thus able to describe the influence of microstructure on conversion vs. time behaviour quite well. The best-fit value for $D_{\mathrm{s}}$ amounts to $2.6 \times 10^{-13} \mathrm{~m}^{2} \mathrm{~s}^{-1}$ for both limestone types. When calculating the product layer diffusion fits using eqn. (16), the $k_{\mathrm{c}}$ value was fixed at $1 \mathrm{~m} \mathrm{~s}^{-1}$. The resulting $\psi$ values are $3 \times 10^{4}$ or larger (depending on grain size; see eqn. (12)), indicating that product layer diffusion is indeed the governing mechanism in these fits.

The obtained best-fit value of $D_{\mathrm{s}}$ appears somewhat low when compared with values used in previous 

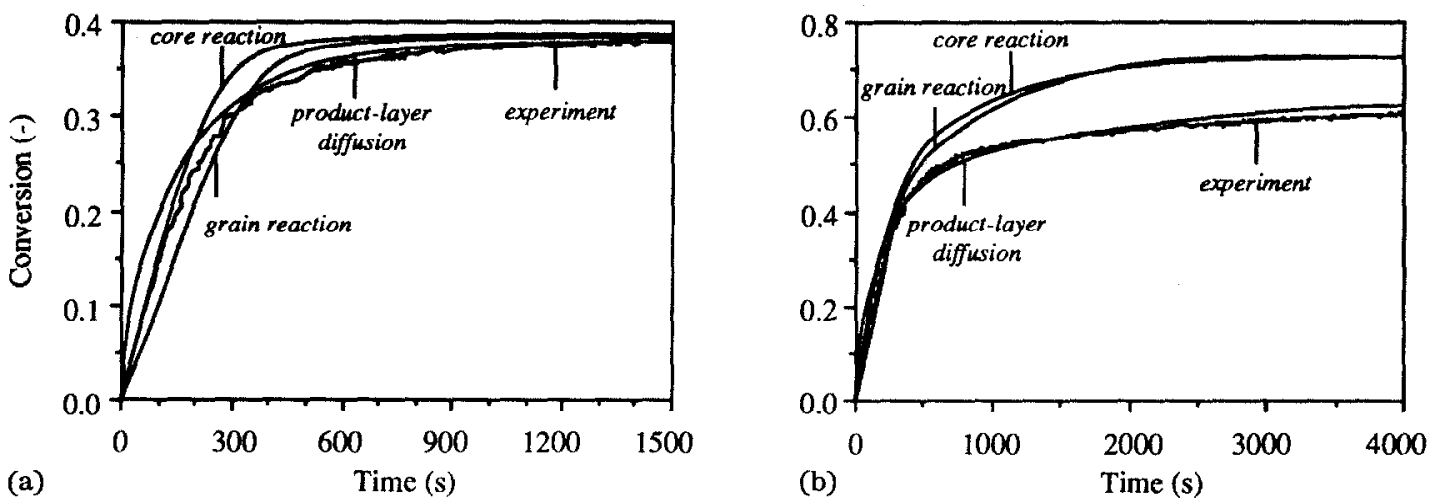

Fig. 7. Measured and calculated conversion $v s$. time curves for the sulphation of (a) Wülfrath and (b) Lhoist agglomerate particles $\left(D_{\mathrm{s}}=2.6 \times 10^{-13} \mathrm{~m}^{2} \mathrm{~s}^{-1}, k_{\mathrm{c}}=5.3 \times 10^{-5} \mathrm{~m} \mathrm{~s}^{-1}, k_{\mathrm{g}}=3.0 \times 10^{-5} \mathrm{~m} \mathrm{~s}^{-1}\right)$.

modelling efforts. Marsh and Ulrichson [21] recorded values ranging from $6.0 \times 10^{-13}$ to $10^{-9} \mathrm{~m}^{2} \mathrm{~s}^{-1}$. These values were derived from experiments carried out by several investigators at temperatures between 650 and $980^{\circ} \mathrm{C}$. Borgwardt and Bruce [22] derived values of $D_{\mathrm{s}}$ at various temperatures ranging from 800 to $1125^{\circ} \mathrm{C}$. They reported an activation energy of $153 \mathrm{~kJ} \mathrm{~mol}^{-1}$. Using this number, our $D_{\mathrm{s}}$ value of $2.6 \times 10^{-13} \mathrm{~m}^{2} \mathrm{~s}^{-1}$ at $700{ }^{\circ} \mathrm{C}$ would yield a value of $3.3 \times 10^{-12} \mathrm{~m}^{2} \mathrm{~s}^{-1}$ at $850{ }^{\circ} \mathrm{C}$, which is in between the data measured by Hartman and Coughlin [3] $\left((0.6-0.86) \times 10^{-12} \mathrm{~m}^{2} \mathrm{~s}^{-1}\right)$ and those measured by Borgwardt [23] $\left(15 \times 10^{-12} \mathrm{~m}^{2} \mathrm{~s}^{-1}\right)$.

The fits for core reaction limitation and grain reaction limitation in Fig. 7 are less accurate. For the Wülfrath particles reasonable fits can still be obtained using a $k_{\mathrm{c}}$ value of $5.3 \times 10^{-5} \mathrm{~m} \mathrm{~s}^{-1}$ (at a fixed $D_{\mathrm{s}}$ value of $10^{13} \mathrm{~m}^{2} \mathrm{~s}^{-1}$, resulting in $\psi$ values smaller than $10^{-24}$, indicating strong core reaction limitation) or a $k_{\mathrm{g}}$ value of $3.0 \times 10^{-5}$ respectively. However, if the same $k_{\mathrm{c}}$ or $k_{\mathrm{g}}$ value is used for the Lhoist agglomerate particles, very bad fits are obtained. Therefore the conclusion that product layer diffusion governs the sulphation rate seems to be justified. This conclusion is supported by the experiences of other researchers. A possible mechanism in which the process of product layer diffusion is rate controlling was proposed by Borgwardt et al. [24].

1. $\mathrm{SO}_{2}+\frac{1}{2} \mathrm{O}_{2} \rightarrow \mathrm{SO}_{3}$ (gas phase reaction).

2. $\mathrm{SO}_{3}+\mathrm{O}^{2-} \rightarrow \mathrm{SO}_{4}{ }^{2-}$ (grain reaction).

3. $\mathrm{SO}_{4}{ }^{2-} / \mathrm{O}^{2-}$ counterdiffusion (product layer diffusion - rate controlling).

$$
\text { 4. } \mathrm{SO}_{4}{ }^{2-}+\mathrm{CaO} \rightarrow \mathrm{CaSO}_{4}+\mathrm{O}^{2-} \text { (core reaction). }
$$

\subsection{Sulphidation}

Figure 8 shows that the Lhoist agglomerate as well as the Wülfrath particles can be sulphided completely. Pore blocking does not occur because of the relatively small difference between the molar volumes of calcium oxide and calcium sulphide $\left(1.68 \times 10^{-5}\right.$ and $2.76 \times 10^{-5} \mathrm{~m}^{3} \mathrm{~mol}^{-1}$ respectively).

Roughly considered, reasonable fits have been obtained for core reaction limitation and grain reaction limitation. However, upon close inspection it seems that fitting based on the assumption of core reaction limitation $\left(k_{\mathrm{c}}=1.0 \times 10^{-4} \mathrm{~m} \mathrm{~s}^{-1}\right.$, $\psi<10^{-22}$ ) is slightly better, especially for the Wülfrath particles. This would lead to the conclusion that some corc reaction controls the sulphidation rate at the applied temperature of $700{ }^{\circ} \mathrm{C}$.

Borgwardt et al. [25] also investigated the mechanism of the reaction between calcium oxide and hydrogen sulphide. They varied the specific surface area of calcined limestone particles by controlled sintering. In the grain theory this specific surface area is related to the initial grain radius (it is assumed that all grains have the same radius):

$R_{\mathrm{o}}=\frac{3}{A \rho_{\text {sol, reac }}}$

According to this relationship, Borgwardt et al. [25] implicitly varied the initial grain radius $R_{0}$ by varying the specific surface area. From eqn. (16) it follows that the time needed to reach a certain degree of conversion is proportional to $R_{\mathrm{o}}$ in the case of core reaction limitation and proportional to $R_{\mathrm{o}}{ }^{2}$ in the case of product layer diffusion limitation. Borgwardt et al. [25] found that the time needed to reach $70 \%$ conversion is proportional to $R_{0}{ }^{2.3}$ and concluded that product layer diffusion governs the sulphidation rate. This conclusion is in contrast with the indication of core-reaction-controlled sulphidation provided by the present study. A possible explanation for this difference may be that Borgwardt et al. [25], while sintering their limestone particles, 


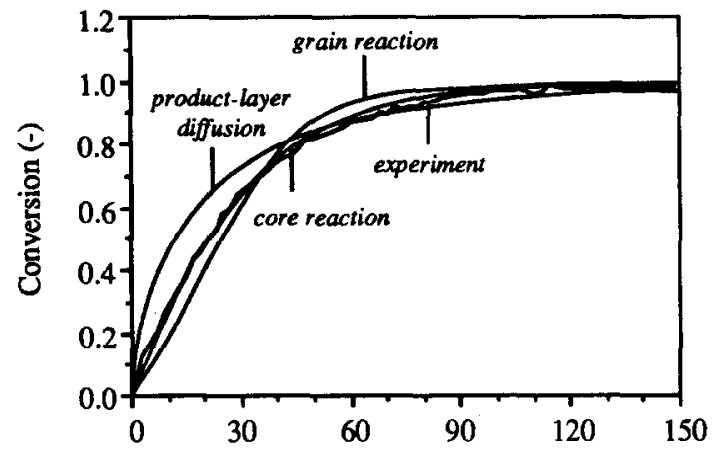

(a)

Time (s)

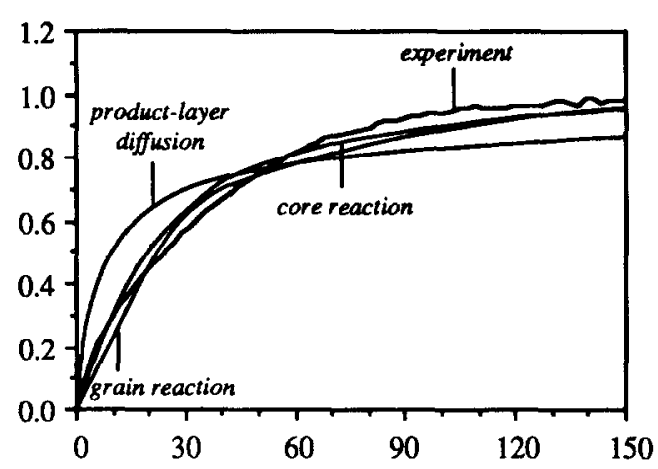

(b)

Time (s)

Fig. 8. Measured and calculated conversion vs. time curves for the sulphidation of (a) Wülfrath and (b) Lhoist agglomerate particles $\left(D_{\mathrm{s}}=1.0 \times 10^{-12} \mathrm{~m}^{2} \mathrm{~s}^{-1}, k_{\mathrm{c}}=1.0 \times 10^{-4} \mathrm{~m} \mathrm{~s}^{-1}, k_{\mathrm{g}}=6.0 \times 10^{-5} \mathrm{~m} \mathrm{~s}^{-1}\right)$.

not only changed the initial grain size but also the reactivity at the core surface. It is quite feasible that the value of $k_{c}$ decreases with increasing sintering time, e.g. because a more perfect (and therefore less reactive) calcium oxide lattice structure is formed. This seems to be confirmed by the fact that the sulphidation rate observed in this study is considerably higher than expected from the study of Borgwardt et al. [25]. On assuming product layer diffusion to be the rate-controlling mechanism, a $D_{\mathrm{s}}$ value of $1.0 \times 10^{-12} \mathrm{~m}^{2} \mathrm{~s}^{-1}$ is found by fitting the present experimental results. This value is much higher than the one derived from the work of Borgwardt et al. [25] $\left(D_{\mathrm{s}}=3.6 \times 10^{-14} \mathrm{~m}^{2} \mathrm{~s}^{-1}\right.$ at $700{ }^{\circ} \mathrm{C}$ ).

Borgwardt et al. [25] proposed the following sulphidation mechanism.

1. $\mathrm{H}_{2} \mathrm{~S}+\mathrm{O}^{2-} \rightarrow \mathrm{H}_{2} \mathrm{O}+\mathrm{S}^{2-}$ (grain reaction).

2. $\mathrm{S}^{2-} / \mathrm{O}^{2-}$ counterdiffusion (product layer diffusion).

3. $\mathrm{S}^{2-}+\mathrm{CaO} \rightarrow \mathrm{CaS}+\mathrm{O}^{2-}$ (core reaction).

They suggest that product layer diffusion is rate controlling. According to our findings, it is more likely that the core reaction is rate controlling.

A future paper will deal in more detail with the kinetics and mechanism of calcium oxide sulphidation.

\subsection{Regeneration}

Figure 9 shows the measured and calculated bestfit conversion $v s$. time curves for the regeneration of sulphided Lhoist dolomite particles. Despite the large difference in the molar volumes of calcium sulphide and calcium carbonate $\left(2.76 \times 10^{-5}\right.$ and $3.69 \times 10^{-5} \mathrm{~m}^{3} \mathrm{~mol}^{-1}$ respectively), full conversion can be obtained owing to the presence of inert magnesium oxide.

By far the best fit is obtained when grain reaction limitation is assumed. The best-fit value of $k_{\mathrm{g}}$

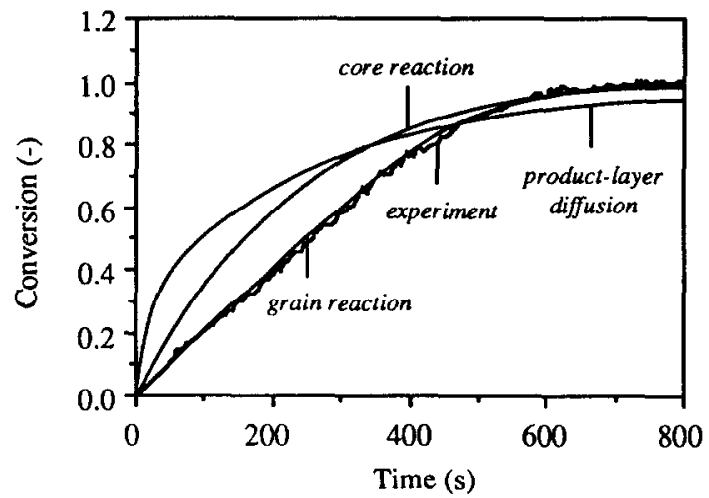

Fig. 9. Measured and calculated conversion vs. time curves for the regeneration of sulphided Lhoist dolomite particles $\left(D_{\mathrm{s}}=1.0 \times 10^{-17} \mathrm{~m}^{5} \mathrm{~mol}^{-1} \mathrm{~s}^{-1}, k_{\mathrm{c}}=1.5 \times 10^{-9} \mathrm{~m}^{4} \mathrm{~mol}^{-1} \mathrm{~s}^{-1}\right.$, $k_{\mathrm{g}}=7.5 \times 10^{-10} \mathrm{~m}^{4} \mathrm{~mol}^{-1} \mathrm{~s}^{-1}$ ).

amounts to $7.5 \times 10^{-10} \mathrm{~m}^{4} \mathrm{~mol}^{-1} \mathrm{~s}^{-1}$. Although some studies were performed previously [26, 27], the precise mechanism of the regeneration reaction has not yet been established. Huang et al. [27] suggest that product layer diffusion might be rate controlling but do not present any evidence for this.

A reaction mechanism in which the grain reaction is rate controlling might be as follows.

1. $\mathrm{CO}_{2}+\mathrm{H}_{2} \mathrm{O}+\mathrm{S}^{2-} \rightarrow \mathrm{CO}_{3}{ }^{2-}+\mathrm{H}_{2} \mathrm{~S}$ (grain reaction - rate controlling).

2. $\mathrm{CO}_{3}{ }^{2-} / \mathrm{S}^{2-}$ counterdiffusion (product layer diffusion).

3. $\mathrm{CO}_{3}{ }^{2-}+\mathrm{CaS} \rightarrow \mathrm{CaCO}_{3}+\mathrm{S}^{2-}$ (core reaction).

A future paper will deal with the kinetics and mechanism of the regeneration reaction of sulphided dolomite with mixtures of carbon dioxide and steam in more detail.

\subsection{Grain size distribution vs. single grain size}

It is interesting to see whether the effort of taking the grain size distribution into account results in 
a significantly better description of the conversion vs. time behaviour. Therefore GSD fits were prepared with a varying number of grain size intervals: one, two and the full number of intervals. Figure 10 shows the results for the best-fit mechanisms obtained for sulphation, sulphidation and regeneration. The quality of the fits does indeed increase with increasing number of grain size intervals, especially in the case of product layer diffusion and grain reaction limitation.
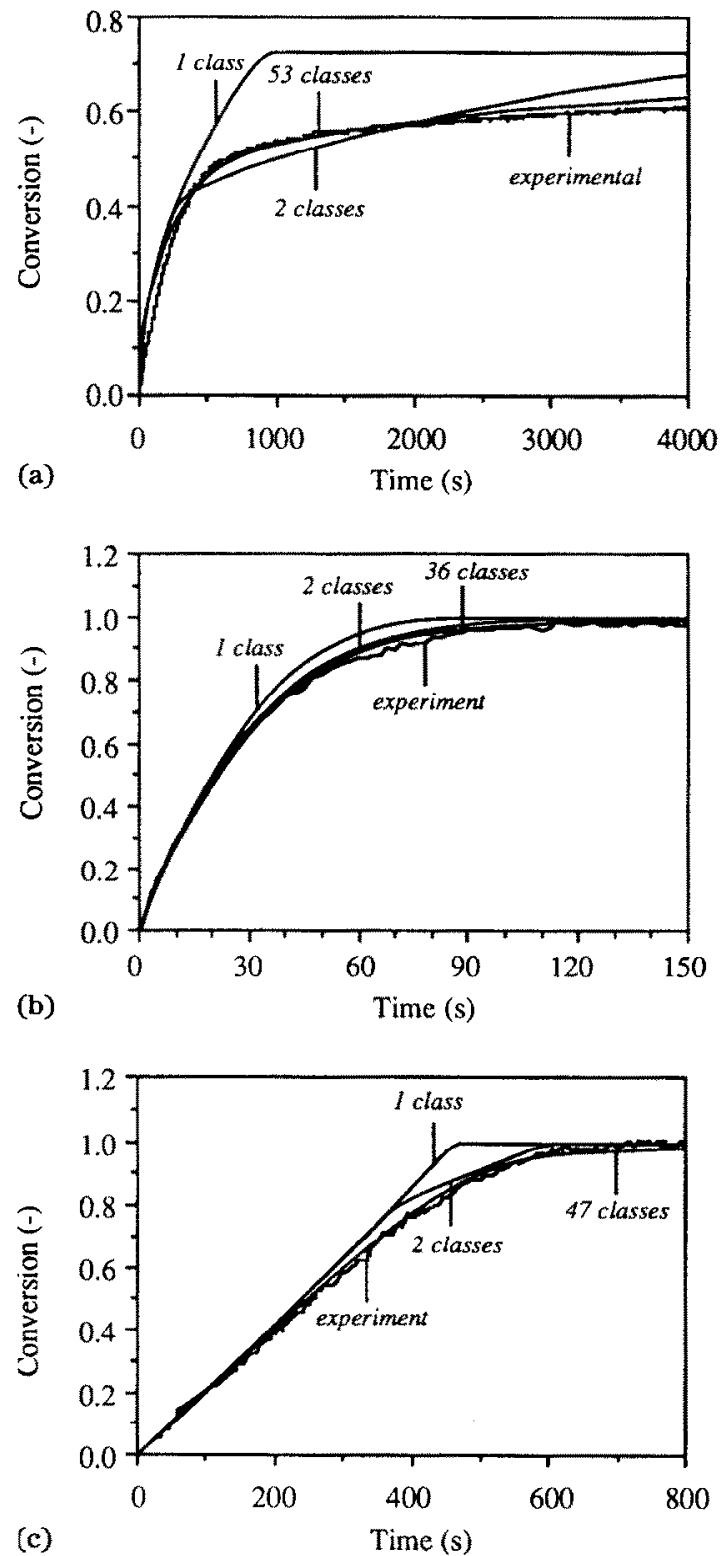

Fig. 10. Measured and calculated conversion vs. time curves for one, two and the full number of pore-grain size intervals: (a) product layer diffusion fits for the sulphation of agglomerated Lhoist limestone $\left(D_{\mathrm{s}}=2.6 \times 10^{-13} \mathrm{~m}^{2} \mathrm{~s}^{-1}\right)$; (b) core reaction its for the sulphidation of Wülfrath limestone $\left(k_{\mathrm{c}}=1.0 \times 10^{-4}\right.$ $\mathrm{m} \mathrm{s}^{-1}$ ); (c) grain reaction fits for the regeneration of sulphided Lhoist dolomite $\left(k_{\mathrm{g}}=7.5 \times 10^{-10} \mathrm{~m}^{4} \mathrm{~mol}^{-1} \mathrm{~s}^{-1}\right)$.

\section{Conclusions}

A grain size distribution model was developed to describe the kinetics of non-catalytic reactions between a reactant gas and a porous solid. The evolution of the solid microstructure during conversion and its influence on reactivity is explicitly taken into account. A measurable pore-to-sphere factor is used to transform the pore size distribution obtained from mercury porosimetric measurements into a grain size distribution. The applicability of this method to micro grains was verified experimentally.

The proposed model was tested by comparing the calculated conversion vs. time behaviour with the measured behaviour during the sulphation and sulphidation of two types of limestone particles of quite different microstructures as well as during the regeneration of sulphided dolomite. The model was applied successfully to determine the mechanisms of the gas-solid reactions involved. Under the applied experimental conditions, sulphation appeared to be governed by product layer diffusion, sulphidation by some core reaction and regeneration by some grain reaction.

\section{Acknowledgments}

This investigation was supported by the Directorate-General XII of the European Communities, TNO-IMET and NOVEM B.V. of the Netherlands. The authors acknowledge H.A. Akse, D.W.F. Brilman, J. de Haan, J. Klaus, H. Nijmeijer and A.G. Steghuis for their assistance in the experimental work and the limestone supply companies Rheinische Kalksteinwerke GmbH (Wülfrath, Germany) and Lhoist Group (Ottignies-Louvain-la-Neuve, Belgium) for their cooperation.

\section{References}

1 S. Yagi and D. Kunii, Proc. 5th Symp. (Int.) on Combustion 1955, Reinhold, New York, p. 231.

2 J. Szekely and J.W. Evans, Chem. Eng. Sci., 25 (1970) 1091.

3 M. Hartman and W.R. Coughlin, AIChE J., 22 (1976) 490.

4 C. Georgakis, C.W. Chang and J. Szekely, Chem. Eng. Sci, 34 (1979) 1072.

5 J. Szekely and M. Propster, Chem. Eng. Sci., 30 (1975) 1049.

6 K. Dam-Johansen, P.F.B. Hansen ard K. Østergaard, Chem. Eng. Sci., 46 (1991) 847.

7 P.V. Ranade and D.P. Harrison, Chem. Eng. Sci, 36 (1981) 1079. 
8 E.A. Efthimiadis and S.V. Sotirchos, Chem. Eng. Sci., 48 (1993) 1201.

9 P.A. Ramachandran and J.M. Smith, AIChE J., 23 (1977) 353.

10 G.R. Gavalas, AIChE J., 26 (1980) 577.

11 S.K. Bhatia and D.D. Perlmutter, AlChE J., 26 (1980) 379.

12 S.K. Bhatia, AIChE .I., 31 (1985) 642.

13 S.E. Zarkanitis, E.A. Efthimiadis and S.V. Sotirchos, Chem. Eng. Sci., 45 (1990) 2761.

14 S. Reyes and K.F. Jensen, Chem. Eny. Sci., 42 (1987) 565.

15 S.V. Sotirchos and S.E. Zarkanitis, AIChE J., 35 (1989) 1137.

16 J.Y. Park and O. Levenspiel, Chem. Eng. Sci., 30 (1975) 1207.

17 H.P. Tseng and T.F. Edgar, Fuel, 68 (1989) 114.

18 R.H. Borgwardt and R.D. Harvey, Environ. Sci. Technol, 6 (1972) 350.

19 L.K. Frevel and L.J. Kressley, Anal. Chem., 35 (1963) 1492.

20 B. Kamphuis and U. Spitsbergen, in P.F. Sens and J.K. Wilkinson (eds.), Flue Gas and Fly Ash, Elsevier Applied Science, London, 1989.

21 D.W. Marsh and D.L. Ulrichson, Chem. Eng. Sci., 40 (1985) 123.

22 R.H. Borgwardt and K.R. Bruce, AIChE J., 32 (1984) 239.

23 R.H. Borgwardt, Environ. Sci. Technol., 4 (1970) 59.

24 R.H. Borgwardt, K.R. Bruce and J. Blake, Proc. Ist Joint Symp. on Dry $\mathrm{SO}_{2}$ and Simultaneous $\mathrm{SO}_{2} / \mathrm{NO}_{x}$ Control Techniques, San Diego, CA, USA, 1984.

25 R.H. Borgwardt, N. Roache and K.R. Bruce, Environ. Prog., 3 (1984) 129.

26 C.C. Sun, E.P. O'Neill and D.L. Keairns, Thermochim. Acta, 26 (1978) 283.

27 C.S. Huang, F.H. Rogan and L. Kun, AIChE Symp. Ser., 77 (1981) 1 .

\section{Appendix A: Nomenclature}

$A \quad$ specific surface area $\left(\mathrm{m}^{2} \mathrm{~kg}^{-1}\right)$

$C_{\mathrm{c}} \quad$ concentration at core surface $\left(\mathrm{mol} \mathrm{m} \mathrm{m}^{-3}\right)$

$C_{\mathrm{g}}$ concentration at grain surface $\left(\mathrm{mol} \mathrm{m}^{-3}\right)$
$D_{\mathrm{s}} \quad$ product layer diffusivity $\left(\mathrm{m}^{2} \mathrm{~s}^{-1}\right.$ or $\mathrm{m}^{5}$ $\mathrm{mol}^{-1} \mathrm{~s}^{-1}$ )

$F \quad$ ratio of grain radius and pore radius (poreto-sphere factor)

$k_{\mathrm{c}} \quad$ reaction rate constant of core reaction (m $\mathrm{s}^{-1}$ or $\mathrm{m}^{4} \mathrm{~mol}^{-1} \mathrm{~s}^{-1}$ )

$k_{\mathrm{B}} \quad$ reaction rate constant of grain reaction (m $\mathrm{s}^{-1}$ or $\mathrm{m}^{4} \mathrm{~mol}^{-1} \mathrm{~s}^{-1}$ )

$K \quad$ expansion factor

$K_{\mathrm{c}} \quad$ overall reaction rate constant (core reaction limitation) $\left(\mathrm{s}^{-1}\right)$

$K_{\mathrm{g}}$ overall reaction rate constant (grain reaction limitation) $\left(\mathrm{s}^{-1}\right)$

$K_{\mathrm{p}} \quad$ overall reaction rate constant (productlayer diffusion limitation $\left(\mathrm{s}^{-1}\right)$

$N \quad$ number of pore and grain size classes

$N_{\mathrm{o}} \quad$ initial concentration of solid reactant in grains $\left(\mathrm{mol} \mathrm{m}{ }^{-3}\right)$

$P \quad$ initial weight fraction of solid reactant in grain (purity)

$r \quad$ radius $(\mathrm{m})$

$R_{\mathrm{c}} \quad$ radius of unreacted core (m)

$R_{\mathrm{g}} \quad$ radius of (partly converted) grain (m)

$R_{\mathrm{o}} \quad$ initial radius of grain (m)

$R_{\mathrm{p}} \quad$ pore radius (m)

$V_{\mathrm{p}} \quad$ specific pore volume $\left(\mathrm{m}^{3} \mathrm{~kg}^{-1}\right)$

$V_{\text {sol, prod }}$ molar volume of solid product $\left(\mathrm{m}^{3} \mathrm{~mol}^{-1}\right)$

$V_{\text {sol, reac }}$ molar volume of solid reactant $\left(\mathrm{m}^{3} \mathrm{~mol}^{-1}\right)$

$X$ conversion of a grain (or particle)

$X_{\max } \quad$ maximum attainable conversion of a grain (or particle)

\section{Greek letters}

$\nu_{i} \quad$ fraction of grains with initial radius $R_{\mathrm{o}, i}$

$\rho_{\text {sol, reac }}$ density of solid reactant $\left(\mathrm{kg} \mathrm{m}^{-3}\right)$

$\psi$ ratio of $K_{\mathrm{c}}$ and $K_{\mathrm{p}}$ 